\title{
Optimal timing of contrast-enhanced computed tomography in an evaluation of severe acute pancreatitis-associated complications
}

\author{
HUALI HUANG ${ }^{1 *}$, WENJING CHEN ${ }^{2 *}$, GUODU TANG $^{1}$, ZHIHAI LIANG $^{1}$, MENGBIN QIN $^{3}$, \\ MINZHEN QIN $^{1}$, YONGFENG TANG ${ }^{1}$, HEPING QIN $^{1}$ and RENJIE CHANG ${ }^{1}$
}

${ }^{1}$ Department of Gastroenterology, The First Affiliated Hospital of Guangxi Medical University, Nanning, Guangxi 530021; ${ }^{2}$ Department of Gastroenterology of The First People's Hospital of Nanning City,

The Fifth Affiliated Hospital of Guangxi Medical University, Nanning, Guangxi 530022; ${ }^{3}$ Department of Gastroenterology,

The Second Affiliated Hospital of Guangxi Medical University, Nanning, Guangxi 530007, P.R. China

Received October 16, 2018; Accepted March 12, 2019

DOI: $10.3892 / \mathrm{etm} .2019 .7700$

\begin{abstract}
Dynamic contrast-enhanced computed tomography (CECT) has been used previously to evaluate severe acute pancreatitis (SAP)-associated complications. However, optimal time points of CECT have not yet been established. The present study aimed to determine optimal timings for CECT to be undertaken for patients with SAP. The results of CECT from 309 patients with SAP, who were classified into either infected or non-infected SAP groups, were retrospectively analyzed. The severity and alterations in the periods within $72 \mathrm{~h}$ to $>4$ weeks of SAP onset were also assessed. In the analysis of the disease severity and changes, acute peripancreatic fluid collection was detected, where the number of areas increased within 1 week of SAP onset but decreased within 4 weeks and longer. However, no significant differences were observed between the infected and non-infected groups. The acute necrotic collection (ANC) areas were $\leq 30 \%$ of the area of the pancreas, with significantly more ANC areas and pancreatic necrosis in the infected SAP group compared with the non-infected SAP group at a time interval of $>4$ weeks. The exudation of pleural effusion (PE) was elevated within 1 week, but decreased within 2 weeks and longer. The difference in the alteration of the exudation of PE was not statistically different between the two groups. In conclusion, the results suggest that the period between $72 \mathrm{~h}$ and 1 week of SAP onset is optimal timing of CECT to assess SAP-associated complications, particularly for infected SAP patients.
\end{abstract}

Correspondence to: Dr Guodu Tang, Department of Gastroenterology, The First Affiliated Hospital of Guangxi Medical University, 6 Shuangyong Road, Nanning, Guangxi 530021, P.R. China E-mail: tguodu02@126.com

${ }^{*}$ Contributed equally

Key words: acute pancreatitis, severe acute pancreatitis, dynamic contrast-enhanced computed tomography, pancreatic infection

\section{Introduction}

Acute pancreatitis (AP) and AP-associated complications are serious clinical conditions with a high mortality, ranging from 4-10\% (1-7). It is estimated that the global annual prevalence of this disease is $13-45$ cases per 100,000 individuals (8). A study performed in the United States has reported that 300,000 of patients with AP were admitted to hospitals each year (9). Of these, 15-20\% developed life-threatening severe acute pancreatitis (SAP) due to local and systemic complications (10) that were mainly characterized by the presence of persistent organ failure (organ failure that lasts for $\geq 2$ days) (11). In the United Kingdom alone, AP accounts for $\sim 20,000$ hospital admissions per year (1). The manifestations of AP can vary, ranging from local to serious systemic inflammation, including organ failure (12). AP is further complicated by necrosis of the pancreatic parenchyma, with or without involving the peripancreatic tissues in an estimated $20-30 \%$ of patients with AP $(12,13)$, with historically high morbidity $(34-95 \%)$ and mortality $(2-39 \%)(14,15)$.

CT scans are important tools that detect complications associated with AP (16). Dynamic contrast-enhanced computed tomography (CECT) has emerged as a standard imaging technique for the evaluation and grading of complications following AP. Bollen et al (16) demonstrated that the sensitivity and specificity of the modified CT severity index (MCTSI) were 78 and $81 \%$, respectively in examinations of AP-associated complications with CECT. In a study of MCTSIs for the assessment of AP severity, the MCTSI exhibited $61 \%$ sensitivity, $66 \%$ specificity and a positive predictive value of $81 \%$, demonstrating the effectiveness of CECT for the screening of SAP in patients with AP (17). However, the timing of CECT, which has been critical to accurately assess disease severity, is still debated. To the best of our knowledge, until now, the optimal time points to schedule CECT in patients with infected and sterile SAP has not yet been established.

The objective of this study was to determine the optimal timing of CECT. In this retrospective study formed of a total of 309 patients with SAP, the severity of SAP during different time intervals was assessed based on the results of CECT and was determined. The results obtained from the current 
study may provide optimal time points for the use of CECT to evaluate patients with SAP, which may improve accuracy of disease evaluation, avoid unnecessary CECT and promote earlier intervention leading to a better prognosis.

\section{Subjects and methods}

Human subjects and study design. A total of 309 patients with SAP who were admitted to the First Affiliated Hospital of Guangxi Medical University (Nanning, Guangxi, China) between February 2002 and July 2016 were retrospectively enrolled in this observational study. The patients ( 230 males and 79 females; aged $\geq 18$ years) had not been treated with antibiotics prior to hospital admission. The patients who had the following conditions were eventually excluded from this retrospective study: i) An external injury; ii) tumors; iii) autoimmune diseases; iv) diabetes or any other disease such as infections and metabolic disorders that could be associated with SAP; or v) eventual pancreatic surgery. The diagnosis of AP was established by a combination of clinical symptoms, laboratory tests, and abdominal imaging examinations according to the 2012 revision of the Atlanta classification and definitions by international consensus (11). In particular, the diagnosis of AP was established if two of the three following criteria were fulfilled: i) Upper abdominal pain of acute onset, which usually radiates to the back; ii) serum levels of amylase or lipase activity more than three times the upper limit of the normal value, and iii) results from the cross-sectional abdominal imaging consistent with AP (11). SAP is defined by the presence of one incidence of local complication and/or systemic complications with organ failure usually either early or late in the disease (18-20). If more than one organ is persistently affected as part of systemic complications, multiple organ dysfunction syndrome (MODS) or multiple organ failure are termed. The markers for the disease severity in AP are most reliable in organ failure lasting for $>48 \mathrm{~h}(21,22)$. In the modified Marshall scoring system, organ failure is defined by a score of at least 2 for $>48 \mathrm{~h}$ for one or more of the three organ systems $(11,23)$. SAP was classified as Balthazar Computed Tomography grade of D or E (24). The MCTSI was calculated by summing these values, and the total score was subsequently categorized as follows: Mild pancreatitis, MCTSI score of 0-2; moderate pancreatitis, MCTSI score of 4-6; severe pancreatitis, MCTSI score of 8-10 $(11,25)$.

The patients of the current study were classified into two groups: Infected-SAP group $(\mathrm{n}=108 ; 35 \%)$ and the sterile-SAP group $(n=201,65 \%)$. The infected-SAP group was defined as patients that were diagnosed with pancreatic infection based on ongoing symptoms of sepsis and/or a combination of clinical signs $(21,26)$. These clinical signs included systemic inflammatory response syndrome (SIRS), which was defined by the presence of two or more of the following criteria: i) Heart rate $>90$ beats $/ \mathrm{min}$; core temperature $<36^{\circ} \mathrm{C}$ or $>38^{\circ} \mathrm{C}$; ii) white blood cell count $<4,000$ or $>12,000 / \mathrm{mm}^{3}$; respiration $>20$ breaths/min or $\mathrm{PCO}_{2}<32 \mathrm{mmHg}$ (11); iii) pancreatic infection diagnosis with bacteria and/or fungi identified via Gram staining in samples obtained from CT-guided aspiration or peritoneal lavage fluid (22) (Other components of the infection included cholelithiasis, respiratory secretions, and positive urine, blood and microbiological cultures); iv) CT results of extraluminal gas within areas of necrosis in the pancreatic and/or peripancreatic tissues (22); or the operative site or the site around the pancreatic drainage site exhibited pus (5). The sterile SAP group exhibited the following characteristics: i) Signs of peritonitis, ii) the presence or absence of SIRS findings, iii) no evidence of positive microorganism culture, iv) a CT that does not reveal bubbles in the pancreas or peripancreas, and v) no purulent fluid in surgical or abdominal puncture drainage.

The protocol for the present study was reviewed and approved by the Institutional Ethics Committee of the First Affiliated Hospital of Guangxi Medical University (Guangxi, China). The requirement for written informed consent was waived due to the retrospective nature of this study.

CECT. The demographic and clinical characteristics of patients with SAP were assessed, which included SAP etiology, the length of their hospital stay, mortality, complications, Balthazar CT grade (24), MCTSI and detailed CT fluid collections. The aforementioned characteristics were analyzed by reviewing patient medical records. CECT was performed as per routine protocol for all patients using a 64-slice CT scanner (LightSpeed VCT; GE HealthCare), a 128-slice CT scanner (Definition Flash; Siemens Healthcare), and a 256-slice CT scanner (RAVOLUSTION 256; GE Healthcare). The MCTSI was determined within $72 \mathrm{~h}, 1,2,3,4$ weeks and $>4$ weeks from the onset of AP to evaluate the severity of pancreatic inflammation and necrosis. Time was defined as a time-course of the CT results for each patient or simply a sporadic pattern of different patients assessed in different time periods. The onset of AP is defined as the time of onset of abdominal pain, not the time of admission to the hospital (11).

Assessment of the severity of acute pancreatitis. Assessment of the severity of acute pancreatitis included acute peripancreatic fluid collection, acute necrotic collection, and the assessment of the presence of pleural effusion and C-reactive protein (CRP) levels, which were performed according to previous reports $(16,27,28)$. Acute peripancreatic fluid collection (APFC) areas included: i) The areas between the posterior wall of the stomach, small omental bursa and pancreas; ii) the areas between the left anterior kidney and the pancreas; iii) the right kidney front and pancreas; and iv) areas in the paracolonic gutters, the perihepatic and perisplenic and intestinal areas around the upper abdominal cavity. Division classification was level I, 1 area; level II, 2 areas; level III, 3 areas; and level IV, 4 areas. Acute necrotic collection (ANC) area: i) $\leq 30 \%$ of the pancreas; and ii) $>30 \%$ of the pancreas $(16,28)$. The presence of PE was confirmed by CECT. CRP levels were determined using a high-sensitive C-reactive protein Assay Kit (Zhejiang Kuake Biotechnology Co., Ltd.). CRP levels (>150 mg/l) were used for predicting the severity of AP.

Statistical analysis. The data were analyzed using SPSS v22.0 software (IBM Corp.). For categorical variables, $\chi^{2}$ tests were used to determine significant differences whereas one-way analysis of variance followed by Least Significant Difference test was used for continuous variables. Ranked 
Table I. Comparative analysis of demographic characteristics, clinical features and treatments for patients with infected SAP vs. sterile SAP.

\begin{tabular}{|c|c|c|c|c|}
\hline Item & Infection group (\%) & Non-infection group (\%) & All (\%) & P-value \\
\hline Sex & & & & $<0.05$ \\
\hline Male & $65(60.2)$ & $165(82.1)$ & $230(74.3)$ & \\
\hline Female & $43(39.8)$ & $36(17.9)$ & $79(25.7)$ & \\
\hline Causative factors & & & & $<0.05$ \\
\hline Biliary infection & $68(63.0)$ & $0(0.0)$ & $68(22)$ & \\
\hline Alcohol use & $15(13.9)$ & $57(28.4)$ & $72(23.3)$ & \\
\hline Hypertriglyceridemiand cholelithiasis & $10(9.3)$ & $41(20.4)$ & $51(16.5)$ & \\
\hline Unexplained reason & $15(13.9)$ & $103(51.2)$ & $118(38.2)$ & \\
\hline Treatments & & & & $>0.05$ \\
\hline Somatostatin & $104(96.3)$ & $197 / 201(98.0)$ & $301(97.4)$ & 0.37 \\
\hline Antibiotics & $107(99.1)$ & 200/201 (99.5) & $307(99.4)$ & 0.65 \\
\hline Dialysis & $12(11.1)$ & $16 / 201(8.0)$ & $28(9.1)$ & 0.36 \\
\hline Total & $108(100)$ & $201(100)$ & $309(100)$ & \\
\hline
\end{tabular}

variables were analyzed using the Mann-Whitney U rank-sum test. APFC and ANC area grades in the infected and sterile groups were standardized by converting each data point into a $z$-value. All t-test and $z$-test were two-tailed and $\mathrm{P}<0.05$ was considered to indicate a statistically significant difference.

\section{Results}

Characteristics of the study patients. A total of 309 patients with SAP were included in the current retrospective study. The characteristics of patients at the time of hospital administration and overall mortality in the two groups (the infected-SAP group and sterile-SAP group) are presented in Tables I and II. Cases of SAP were predominantly composed of male patients (230 males vs. 79 females; 74.3 vs. $25.7 \%$ ), of which the infected group had 108 patients in total, $60.2 \%$ males and 39.8\% females. The sterile group had 201 patients in total, of which $82.1 \%$ males and $17.9 \%$ females. The two groups exhibited statistically significant sex differences $(\mathrm{P}<0.05)$. The mean ages of the infected-SAP and the sterile-SAP were $50.7 \pm 16.4$ years and $43.6 \pm 12.8$, respectively (data not shown). The overall mortality of patients was $10.7 \%$ $(33 / 309)$, with higher rate of $14.8 \%$ (16/108) observed in the infected group than $8.5 \%(17 / 201)$ in the sterile group that did not reach statistical significance (data now shown). Of the 108 patients in the infected group, 30 developed pancreatic necrosis and had mortality rate of as high as $33.3 \%$ (10/30) (data not shown). Etiological analysis of these patients revealed possible causative factors including heavy alcohol consumption, hyperlipidemia and cholelithiasis. Biliary infection was demonstrated to occur more often in the infected group, while more prevalent alcohol use was exhibited in the sterile group.

The predicted severity of the patients was also evaluated. CRP data were available in 35 patients $(32.4 \%)$ in the infected group and 69 patients $(34.3 \%)$ in the sterile group (data not shown). At $72 \mathrm{~h}$ following the onset of SAP, 16 (14.8\%) and
$36(17.9 \%)$ patients in the infected group and sterile groups respectively had CRP levels higher than $150 \mathrm{mg} / \mathrm{l}$; after $72 \mathrm{~h}$ following the onset of SAP, the numbers increased to 30 $(27.8 \%)$ and $53(26.4 \%)$ respectively in the infected and sterile groups (data not shown). A total of 53/309 (17.2\%) MODS cases occurred in the study patients, with a statistically significant higher percentage of cases in the in the infected group $(26 / 108,24.1 \%)$ vs. the sterile group $(27 / 201,13.4 \%)$ (data now shown). Additionally, the body mass index (BMI) was compared between the groups and no statistically significant differences were found in the infected group $\left(24.6 \pm 4.0 \mathrm{~kg} / \mathrm{m}^{2}\right)$ vs. the sterile group $\left(25.3 \pm 3.7 \mathrm{~kg} / \mathrm{m}^{2}\right)$ (data not shown).

Assessment of acute pancreatitis at different time intervals using different grading systems in the infected and sterile SAP groups. The severity of AP, particularly the proportions of Balthazar Grade E and MCTSI Grade III patients in the infected and sterile groups was compared using the Balthazar and MCTSI grading systems. The classification was based on the results of CECT performed at different time intervals, subdivided in the time periods from $72 \mathrm{~h}$ of onset to $>4$ weeks. As presented in Fig. 1A and B, the infected group exhibited a higher number of Balthazar Grade E and MCTSI Grade III patients compared with the non-infection group. Following four weeks of onset, all patients in the infected group were classified as Balthazar Grade E and MCTSI Grade III (Fig. 1A and B).

CECT results of SAP at different time intervals in the infectedand non-infected SAP groups. The results of CECT undertaken per protocol in the study subjects at different time intervals were analyzed within $72 \mathrm{~h}$, or 1, 2, 3, 4 and $>4$ weeks from the onset of SAP to compare its severity. The groups were classified into four grades based on APFC areas (Grade I, 1 area; Grade II, 2 areas; Grade III, 3 areas; Grade IV, 4 areas) between the infected and sterile groups. Four APFC areas were examined as described in the materials and methods section. APFC 
was primarily observed in four areas of all study subjects and the fluid accumulated 1 week after SAP onset, but reduced 2 weeks later (Fig. 2A, D and E). The CT findings indicated that there were four peripancreatic fluid collection areas and $<30 \%$ pancreatic necrosis at one week from the onset of AP (Fig. 2D). There was no apparent change in the peripancreatic fluid collection areas compared with those at 1 week from the onset of AP, while pancreatic necrosis was reduced in contrast to that at 1 week from the onset of AP (Fig. 2E). No significant differences were observed at indicated time intervals from the onset of SAP between the infected and sterile groups (Table II). In an analysis of acute necrotic collection (ANC) areas, ANC areas were classified into three levels (No ANC area, ANC areas $\leq 30 \%$ of the pancreas and ANC areas $>30 \%$ of the pancreas). It was revealed that the ANCs areas in all patients in the infected and non-infected groups at the indicated time intervals predominantly covered $\leq 30 \%$ of the pancreas and the SAP patients with a higher degree of necrosis were prone to be infected (Fig. 2B and D). The differences between the two groups were statistically significant for within $72 \mathrm{~h}$ and four weeks (Table III). Pleural effusion was detected in all the study subjects of each group in the time periods from $72 \mathrm{~h}$ to $>4$ weeks of onset (Fig. 2C). Furthermore, no significant differences between the infected group and sterile group were observed at any time point (Table IV).

Comparative analysis of alterations in CETC results at different time intervals in the infected and non-infected groups. The differences in CECT results at different time periods from $72 \mathrm{~h}$ to $>4$ weeks of onset between the infected and non-infected groups were subsequently compared. Changes in APFC areas detected via CECT indicated that the number of APFC areas increased within 1 week of onset but reduced within 2 weeks and longer in comparison with the previous CECT (undertaken within 72 h) in all the study subjects (Fig. 3A). Furthermore, differences between the infected and non-infected groups were not statistically different (Table V). It was observed that the majority of ANC areas on CECT increased within 1 week and at $>4$ weeks of SAP onset compared with that of the previous $\mathrm{CT}$ (undertaken within $72 \mathrm{~h}$ ) in all the patients with SAP (Fig. 3B), whereas no significant change was observed between the time intervals within 2 and 4 weeks (Table VI). In addition, the infected group exhibited an increase in the ANC areas at the time intervals of 2,3 and $>4$ weeks and exhibited greater pancreatic necrosis in contrast to the sterile group at 4 weeks (Table VI). The results also revealed that the exudation of PE in each group was increased within 1 week of onset compared with that in the previous CECT results, but declined at time intervals of within 2 weeks and longer in all study subjects (Fig. 3C). Furthermore, the differences between the infected group and non-infected group were not statistically different (Table VII). Notably, 4 of 309 study subjects developed infected pancreatic necrosis, showing bubbles on the CECT imaging (data not shown).

\section{Discussion}

CECT has been widely used as an imaging technique to assess local and systemic complications in patients with AP (16). To date, the appropriate time points for when CECT should 
A

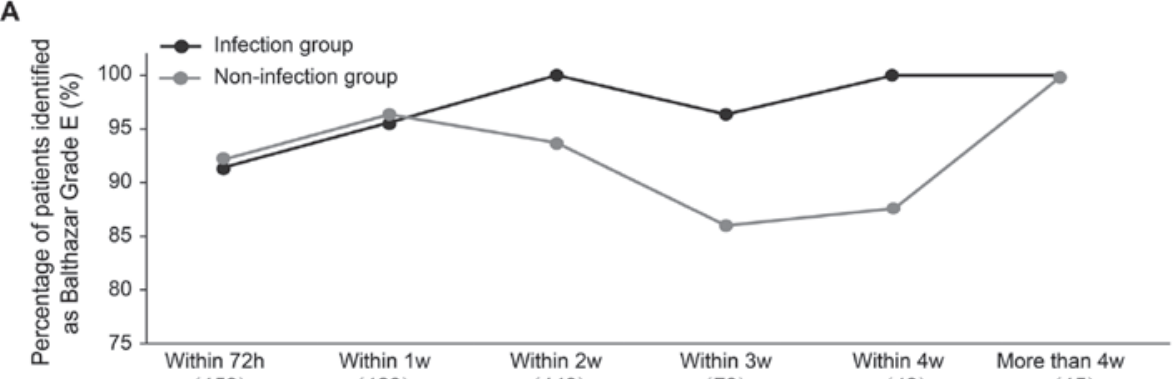

$\begin{array}{lllll}(159) & (128) & (149) & (70) & (43)\end{array}$

Different time intervals (number of patients subjected to CECT at each time interval)

B

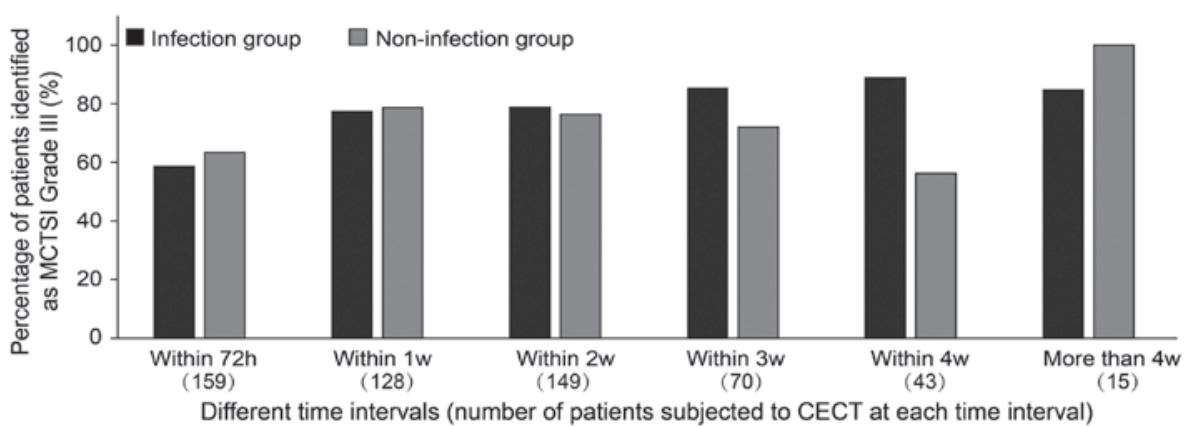

Figure 1. Severity assessment using different grading systems in the infected and non-infected SAP groups. The proportions of the (A) Balthazar Grade E and (B) MCTSI Grade III patients in the infected and non-infected SAP groups were determined based on the results of CECT in different time intervals within $72 \mathrm{~h}$ to $>4$ weeks of SAP onset. SAP, severe acute pancreatitis; MCTSI, modified CT severity index; CECT, contrast-enhanced computed tomography.

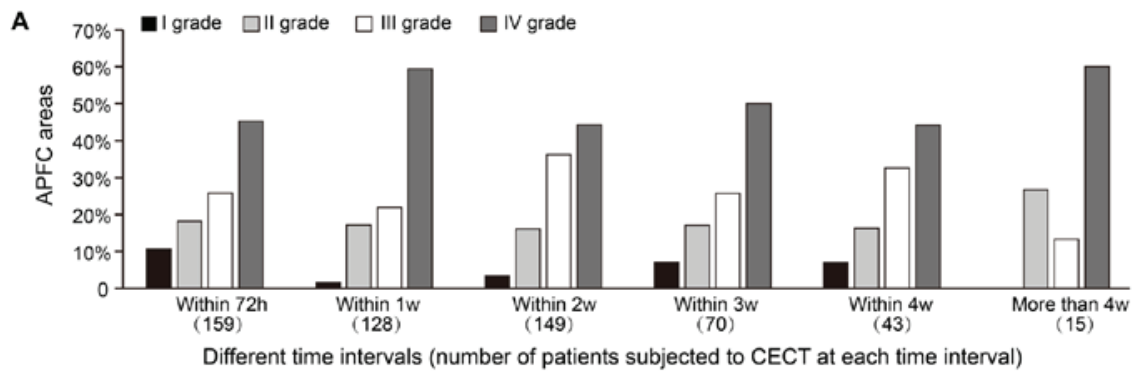

B
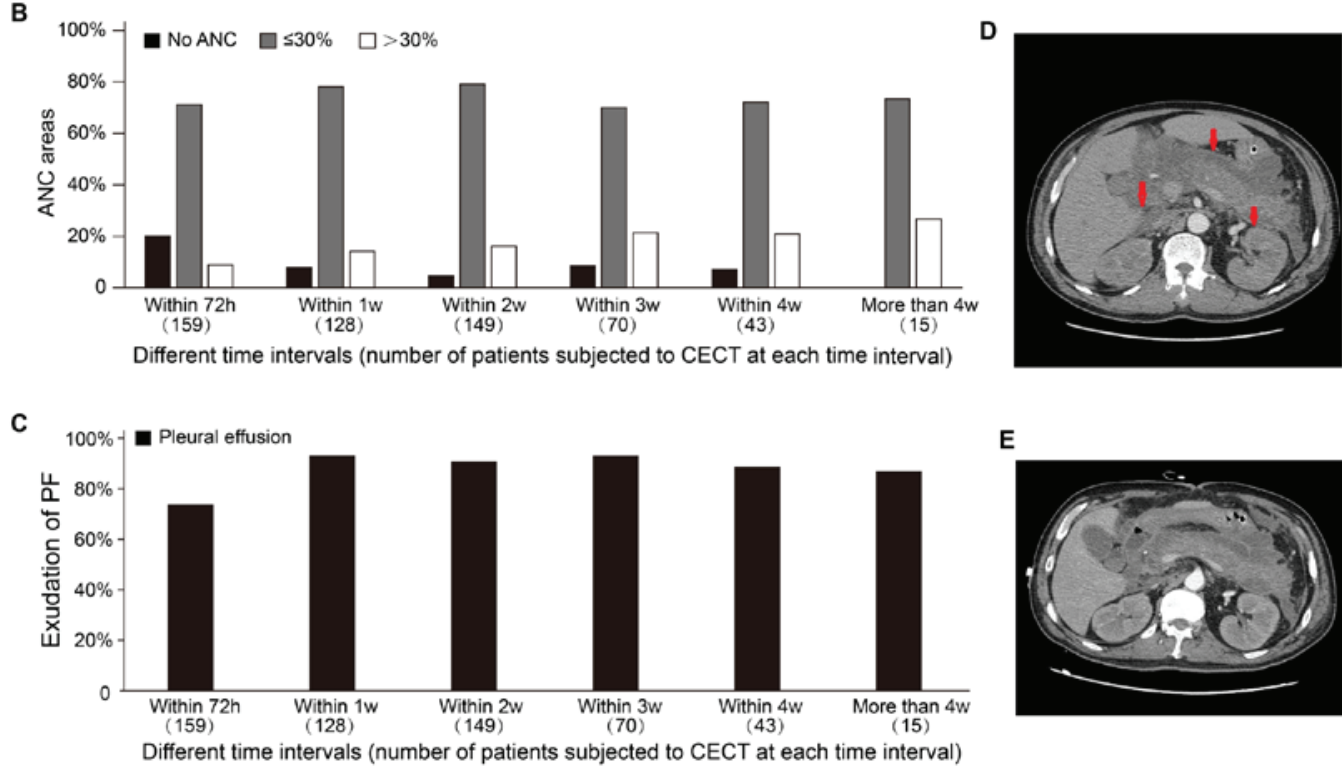

Figure 2. CECT results of the study subjects at different time intervals. (A) APFC, (B) ANC areas, and (C) exudation of PE were assessed according to the CECT findings at different time intervals from $72 \mathrm{~h}$ of SAP onset to $>4$ weeks in all of the study patients. (D) Representative CECT image of a studied patient with AP patient at 1 week from the onset of AP. (E) Representative CECT image of a patient with AP at 2 weeks from the onset of AP. The arrows indicate the increased pleural effusion. APFC, acute peripancreatic fluid collection; ANC, acute necrotic collection; PE, pleural effusion; SAP, severe acute pancreatitis; CECT, contrast-enhanced computed tomography; AP, acute pancreatitis. 
be undertaken to offer an accurate disease evaluation have not been well established in clinical practice $(16,17)$. The present retrospective study of CECT results, severities and their alterations during different time intervals in a total of 309 patients with SAP, who were stratified into infected and non-infected SAP groups. The results demonstrated that APFC was detected in infected and non-infected patients and that the number of areas increased within 1 week of onset but decreased within 2 weeks and beyond in comparison with those at the within $72 \mathrm{~h}$ of onset. However, no significant differences were observed in the two groups. The ANC areas in the two groups were predominantly $\leq 30 \%$ of the pancreas, whereas the infected group exhibited significantly more ANC areas and pancreatic necrosis than non-infected patients at the time interval of $>4$ weeks of onset. In addition, the exudation of PE was increased within 1 week of onset, but on the decline within 2 weeks and beyond in the infected and non-infected groups. However, the observed differences were not statistically different. Furthermore, the present results showed the period between $72 \mathrm{~h}$ and one week of onset was optimal timing of CECT for an evaluation of SAP-associated complications, particularly for infected patients.

The mortality of patients with AP is high, ranging from $\sim 4-10 \%$ and greater in cases of life-threatening severe necrotizing pancreatitis, with an estimated rate of $15-30 \%(1,6,11)$. In the present study, the mortality rate for patients with SAP was as high as $10.7 \%$, which was not significantly correlated with pancreatic infection (data not shown). However, it was observed that the infected-SAP group exhibited a greater severity of infection. In addition, the patients in the infection group were older than those in the non-infection group, indicating a greater possibility of infection in patients with older age (data not shown).

CECT as an imaging modality has been highly recommended in the diagnosis, severity evaluation and morphological classification of SAP $(16,17)$. In addition, it has provided helpful information about fluid collections, thickness and the internal debris of the pancreas $(16,17)$. It is also ideal for guiding percutaneous aspiration and drainage procedures. Furthermore, CECT allows examiners to detect morphological features as a part of SAP diagnosis to determine disease severity $(16,17)$. However, the optimal timing for performing a CECT in patients with suspected SAP remains controversial $(11,16,17)$. It has been demonstrated that CECT scheduled and performed immediately after the onset of symptoms may result in damaging the pancreas and underestimating the disease severity (11). In the present study, patients with SAP $(n=309)$ in the infected and non-infected groups primarily possessed a Balthazar grade of E and a MCTSI grade of III. In addition, APFCs were primarily observed in four areas that did not correlate with pancreatic fluid collection infection. The fluid accumulated one week but reduced after two weeks of SAP onset, which was consistent with previous results that fluid collections were found to develop in the early phase and improved in the late phase of pancreatitis (11). Although ANCs covered $\leq 30 \%$ of all patient pancreases in the current study, patients with SAP and a higher degree of necrosis were more prone to be infected. No change in ANC areas was observed from 2 to 4 weeks of SAP onset. Furthermore, more ANC areas were identified in the infected group compared with the 
Table IV. Total pleural effusion information in the infected and non-infected severe acute pancreatitis groups.

\begin{tabular}{|c|c|c|c|c|c|c|}
\hline \multirow[b]{2}{*}{ Time } & \multicolumn{2}{|c|}{ Infection group } & \multicolumn{2}{|c|}{ Non-infection group } & \multirow[b]{2}{*}{ All } & \multirow[b]{2}{*}{ P-value } \\
\hline & No $(\%)$ & Yes $(\%)$ & No $(\%)$ & Yes $(\%)$ & & \\
\hline Within $72 \mathrm{~h}$ & $17 / 58(29.3)$ & $41 / 58(70.7)$ & 25/101 (24.8) & $76 / 101(75.2)$ & 159 & 0.53 \\
\hline Within 1 week & $3 / 44(6.8)$ & 41/44 (93.2) & $6 / 84(7.1)$ & 78/84 (92.9) & 128 & 0.95 \\
\hline Within 2 weeks & $8 / 52(15.4)$ & $44 / 52(84.6)$ & $6 / 97(6.2)$ & 91/97 (93.8) & 149 & 0.07 \\
\hline Within 3 weeks & $2 / 27(7.4)$ & $25 / 27(92.6)$ & $3 / 43(7)$ & $40 / 43(93)$ & 70 & 0.95 \\
\hline Within 4 weeks & $2 / 27(7.4)$ & $25 / 27(92.6)$ & $3 / 16(18.8)$ & $13 / 16(81.3)$ & 43 & 0.26 \\
\hline$>4$ weeks & $2 / 13(15.4)$ & $11 / 13(84.6)$ & 0 & $2 / 2(100)$ & 15 & 1.00 \\
\hline Pancreatic infection & $1 / 30(3.3)$ & $29 / 30(96.7)$ & & & 30 & N/A \\
\hline
\end{tabular}

N/A, not applicable.

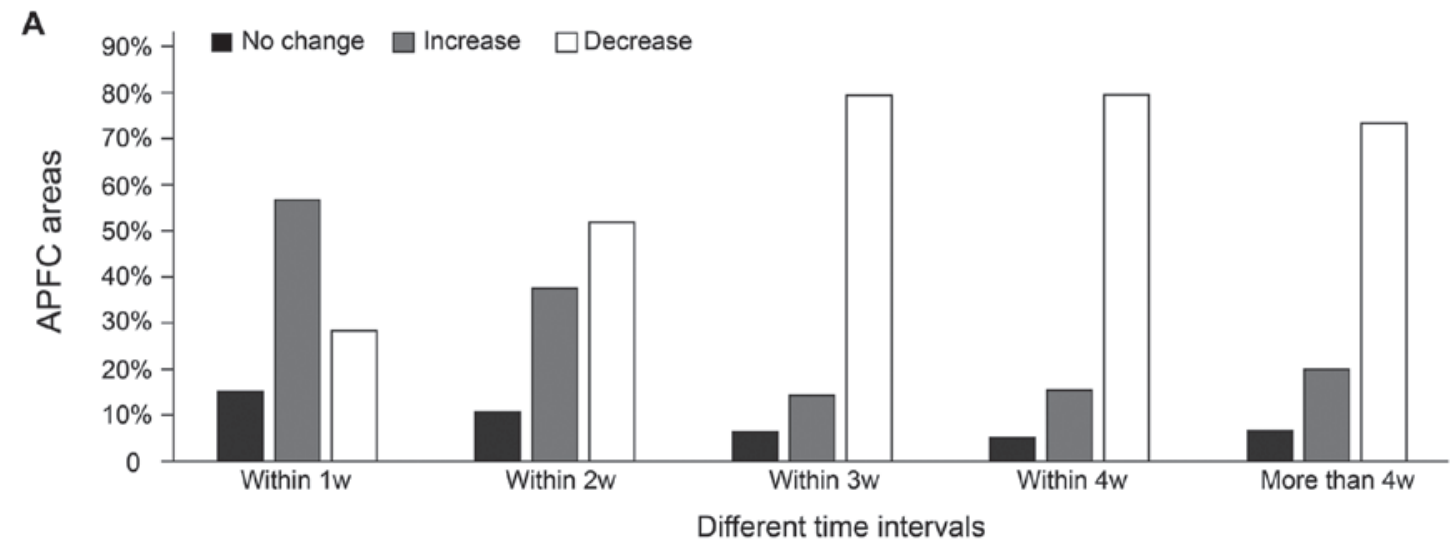

B

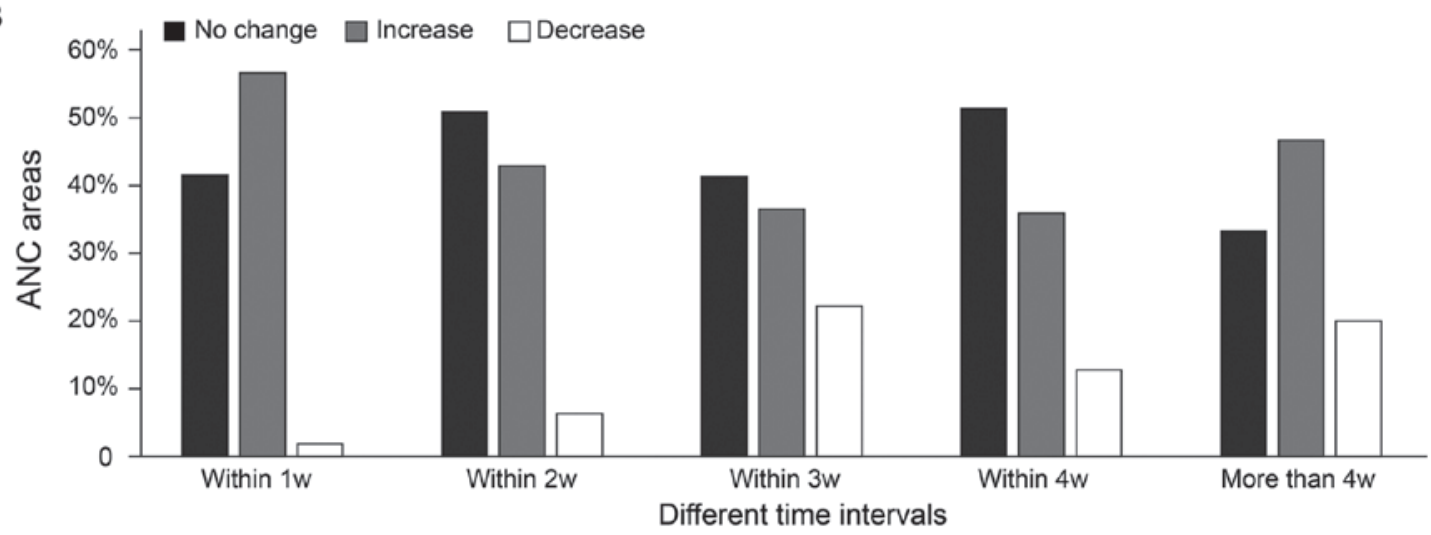

C

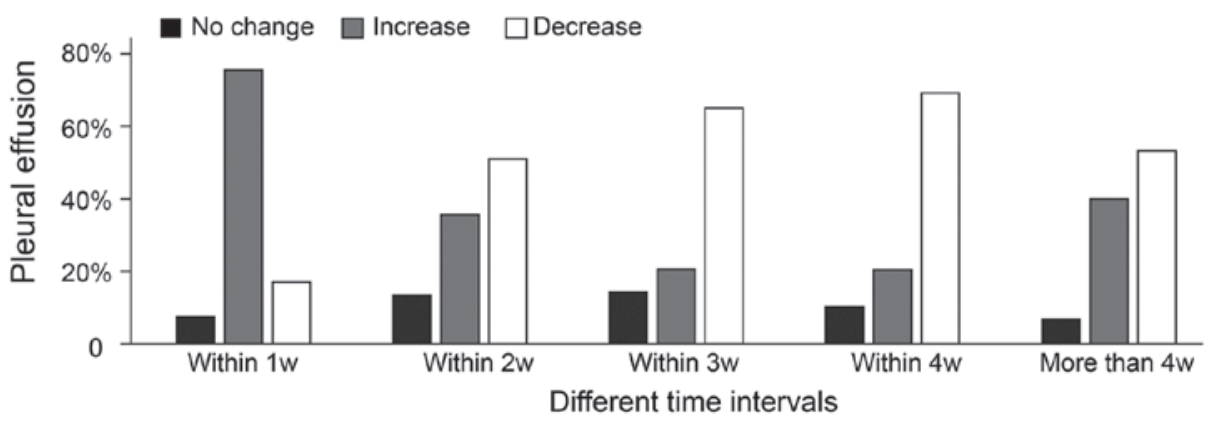

Figure 3. Alterations in CECT results at different time intervals in patients with SAP. The alterations in CECT results at different time periods within $72 \mathrm{~h}$ to $>4$ weeks of acute pancreatitis onset of all patients were compared. Changes in the (A) APFC areas, (B) ANC areas and (C) PE as revealed in all patients via CECT. CECT, contrast-enhanced computed tomography; SAP, severe acute pancreatitis; APFC, acute peripancreatic fluid collection; ANC, acute necrotic collection; PE, pleural effusion. 
Table V. Total acute peripancreatic fluid collection compared with that observed on the previous computed tomography.

\begin{tabular}{|c|c|c|c|c|c|c|c|c|}
\hline \multirow[b]{2}{*}{ Time } & \multicolumn{3}{|c|}{ Infection group } & \multicolumn{3}{|c|}{ Non-infection group } & \multirow[b]{2}{*}{$\mathrm{All}^{\mathrm{a}}$} & \multirow[b]{2}{*}{ P-value } \\
\hline & No change $(\%)$ & Increase (\%) & Decrease $(\%)$ & No change (\%) & Increase $(\%)$ & Decrease $(\%)$ & & \\
\hline Within 1 week & $3 / 16(18.8)$ & $8 / 16(50.0)$ & $5 / 16(31.3)$ & $5 / 37(13.5)$ & $22 / 37(59.5)$ & $10 / 37(27)$ & 53 & 0.8 \\
\hline Within 2 weeks & $5 / 41(12.2)$ & $13 / 41(31.7)$ & 23/41 (56.1) & 7/71 (9.9) & 29/71 (40.8) & 35/71 (49.3) & 112 & 0.62 \\
\hline Within 3 weeks & $2 / 23(8.7)$ & $3 / 23(13.0)$ & $18 / 23(78.3)$ & $2 / 40(5.0)$ & $6 / 40(15.0)$ & $32 / 40(80.0)$ & 63 & 0.84 \\
\hline Within 4 weeks & $2 / 26(7.7)$ & $5 / 26(19.2)$ & $19 / 26(73.1)$ & 0 & $1 / 13(7.7)$ & $12 / 13(92.3)$ & 39 & 0.49 \\
\hline$>4$ weeks & $1 / 13(7.7)$ & 2/13 (15.4) & $10 / 13(76.9)$ & 0 & $1(50)$ & $1(50)$ & 15 & 0.36 \\
\hline
\end{tabular}

${ }^{a}$ The number of patients who had two computed tomography scans for comparative analysis of total acute peripancreatic fluid collection.

Table VI. Total severe acute pancreatitis acute necrotic collection area compared with that observed on the previous computed tomography.

Infection group

Time

Withir

Within 1 week

Within 2 weeks

Within 3 weeks

Within 4 weeks

$>4$ weeks

$5 / 16(31.3)$

(13.5)

$40(5.0)$

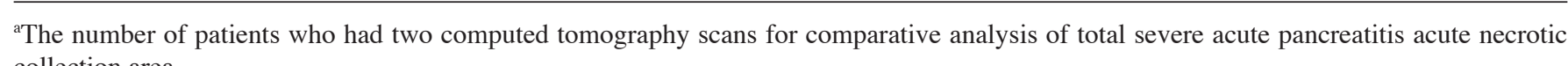
collection area.

Table VII. Total severe acute pancreatitis pleural effusion compared with that of the previous computed tomography.

\begin{tabular}{|c|c|c|c|c|c|c|c|c|}
\hline \multirow[b]{2}{*}{ Time } & \multicolumn{3}{|c|}{ Infection group } & \multicolumn{3}{|c|}{ Non-infection group } & \multirow[b]{2}{*}{$\mathrm{All}^{\mathrm{a}}$} & \multirow[b]{2}{*}{ P-value } \\
\hline & No change $(\%)$ & Increase $(\%)$ & Decrease (\%) & No change $(\%)$ & Increase $(\%)$ & Decrease $(\%)$ & & \\
\hline Within 1 week & $2 / 16(12.5)$ & $11 / 16(68.8)$ & $3 / 16(18.8)$ & $2 / 37(5.4)$ & 29/16 (78.4) & 6/16 (16.2) & 53 & 0.63 \\
\hline Within 2 weeks & 4/41 (9.8) & $14 / 41(34.1)$ & $23 / 41(56.1)$ & $11 / 72(15.5)$ & $26 / 72(36.6)$ & $34 / 72(47.9)$ & 112 & 0.60 \\
\hline Within 3 weeks & $5 / 23(21.7)$ & $5 / 23(21.7)$ & $13 / 23(56.5)$ & $4 / 40(10.0)$ & $8 / 40(20.0)$ & 28/40 (70.0) & 63 & 0.40 \\
\hline Within 4 weeks & $3 / 26(11.5)$ & $7 / 26(26.9)$ & $16 / 26(61.5)$ & $1 / 13(7.7)$ & $1 / 13(7.7)$ & $11 / 13(84.6)$ & 39 & 0.31 \\
\hline$>4$ weeks & $1 / 13(7.7)$ & $6 / 13(46.2)$ & $6 / 13(46.2)$ & 0 & 0 & $2 / 2(100)$ & 15 & 0.36 \\
\hline
\end{tabular}

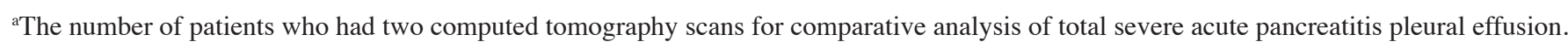

non-infected SAP group, indicating greater levels of necrosis and a higher possibility of infection.

In a previous study, Kayar et al (29) assessed 180 patients with AP and mild pancreatitis with the aim of identifying patients who should be considered for home-monitoring. The results from a previous study indicated that there were no significant alterations in the CT findings of mild AP patients at 12 and $72 \mathrm{~h}$ (29). Another previous study assessed the role of low molecular weight heparin in the management of patients with moderately severe AP (MSAP) (30). Tozlu et al (30) revealed that CECT examination between $72 \mathrm{~h}$ and 1 week is the ideal timeframe to detect MSAP-associated complications. There is a possibility that the severity of SAP may contribute to the different changes of CT results among patients with SAP in the present study.

Despite the effectiveness for CECT in the assessment of SAP-associated complications, it has limitations. For instance, due to the fact that a minimum of 2 weeks was needed for the improvement of liquid accumulation, but not for the improvement in necrosis, CECT may possess a low diagnostic rate of 
pancreatic infection $(16,17,28)$. Furthermore, its specificity and sensitivity are not high $(11,16,17,28)$. Therefore, physicians may identify and evaluate the condition through clinical observations and thus reduce the frequency of CECT, thereby reducing patient costs. The present study has a number of limitations primarily concerned with it being a retrospective study with a relatively small sample size. This may therefore lead to bias in the sample selection of the present study. Therefore, a prospective trial study is required to validate the optimal timings identified in the current study.

In conclusion, the results of the present study demonstrate that CECT monitoring is required for patients with SAP, particularly for the evaluation of SAP-associated complications in infected patients. The present findings also suggest that the time period between $72 \mathrm{~h}$ and 1 week of the disease onset may be optimal timing to use CECT to evaluate SAP-associated complications.

\section{Acknowledgements}

Not applicable.

\section{Funding}

The current study was supported by grants from the National Natural Science Foundation of China (grant no. 1260087), the National Natural Science Foundation of China (grant no. 81560111), and the National Natural Science Foundation of Guangxi Zhuang Autonomous Region (grant no. 2013GXNSFAA019186).

\section{Availability of data and materials}

The datasets used and/or analyzed during the current study are available from the corresponding author on reasonable request.

\section{Authors' contributions}

HLH, WJC and GDT designed the study. HLH, WJC, GDT, ZHL, MBQ, MZQ, YFT, HPQ and RJC collected and analyzed the data. HLH, WJC, GDT and ZHL wrote the manuscript. GDT revised the manuscript critically for intellectual content. All authors gave intellectual input to the study and approved the final version of the manuscript.

\section{Ethics approval and consent to participate}

The study protocol was reviewed and approved by the Institutional Ethic Committee of the First Affiliated Hospital of Guangxi Medical University (Nanning, Guangxi, China). All procedures performed in studies involving human participants were in accordance with the ethical standards of the institutional and national research committee and with the 1964 Helsinki declaration and its later amendments or comparable ethical standards.

\section{Competing interests}

The authors declare that they have no competing interests.

\section{References}

1. Williams JG, Roberts SE, Ali MF, Cheung WY, Cohen DR, Demery G, Edwards A, Greer M, Hellier MD, Hutchings HA, et al: Gastroenterology services in the UK. The burden of disease, and the organisation and delivery of services for gastrointestinal and liver disorders: A review of the evidence. Gut 56 (Suppl 1): S1-S113, 2007.

2. Roberts SE, Williams JG, Meddings D and Goldacre MJ: Incidence and case fatality for acute pancreatitis in England: Geographical variation, social deprivation, alcohol consumption and aetiology-a record linkage study. Aliment Pharmacol Ther 28: 931-941, 2008.

3. Floyd A, Pedersen L, Nielsen GL, Thorladcius-Ussing O and Sorensen HT: Secular trends in incidence and 30-day case fatality of acute pancreatitis in North Jutland County, Denmark: A register-based study from 1981-2000. Scand J Gastroenterol 37: 1461-1465, 2002.

4. Tinto A, Lloyd DA, Kang JY, Majeed A, Ellis C, Williamson RC and Maxwell JD: Acute and chronic pancreatitis-diseases on the rise: A study of hospital admissions in England 1989/90-1999/2000. Aliment Pharmacol Ther 16: 2097-2105, 2002.

5. Goldacre MJ and Roberts SE: Hospital admission for acute pancreatitis in an English population, 1963-98: Database study of incidence and mortality. BMJ 328: 1466-1469, 2004.

6. Frey CF, Zhou H, Harvey DJ and White RH: The incidence and case-fatality rates of acute biliary, alcoholic, and idiopathic pancreatitis in California, 1994-2001. Pancreas 33: 336-344, 2006.

7. Sandzén B, Rosenmüller M, Haapamäki MM, Nilsson E, Stenlund HC and Öman M: First attack of acute pancreatitis in Sweden 1988-2003: Incidence, aetiological classification, procedures and mortality-a register study. BMC Gastroenterol 9: 18, 2009.

8. Yadav D and Lowenfels AB: The epidemiology of pancreatitis and pancreatic cancer. Gastroenterology 144: 1252-1261, 2013.

9. Sandler RS, Everhart JE, Donowitz M, Adams E, Cronin K, Goodman C, Gemmen E, Shah S, Avdic A and Rubin R: The burden of selected digestive diseases in the United States. Gastroenterology 122: 1500-1511, 2002.

10. Forsmark CE and Baillie J; AGA Institute Clinical Practice and Economics Committee; AGA Institute Governing Board: AGA Institute technical review on acute pancreatitis. Gastroenterology 132: 2022-2044, 2007.

11. Banks PA, Bollen TL, Dervenis C, Gooszen HG, Johnson CD, Sarr MG, Tsiotos GG and Vege SS; Acute Pancreatitis Classification Working Group: Classification of acute pancreatitis-2012: Revision of the Atlanta classification and definitions by international consensus. Gut 62: 102-111, 2013.

12. Bradley EL III: A clinically based classification system for acute pancreatitis. Summary of the International Symposium on Acute Pancreatitis, Atlanta, Ga, September 11 through 13, 1992. Arch Surg 128: 586-590, 1993.

13. Beger HG, Rau B, Mayer J and Pralle U: Natural course of acute pancreatitis. World J Surg 21: 130-135, 1997.

14. Baron TH and Morgan DE: Acute necrotizing pancreatitis. N Engl J Med 340: 1412-1417, 1999.

15. Sakorafas GH, Tsiotos GG and Sarr MG: Extrapancreatic necrotizing pancreatitis with viable pancreas: A previously under-appreciated entity. J Am Coll Surg 188: 643-648, 1999.

16. Bollen TL, Singh VK, Maurer R, Repas K, van Es HW, Banks PA and Mortele KJ: Comparative evaluation of the modified CT severity index and CT severity index in assessing severity of acute pancreatitis. AJR Am J Roentgenol 197: 386-392, 2011

17. Jáuregui-Arrieta LK, Alvarez-López F, Cobián-Machuca $H$, Solís-Ugalde J, Torres-Mendoza BM and Troyo-Sanromán R: Effectiveness of the modify tomographic severity index in patients with severe acute pancreatitis. Rev Gastroenterol Mex 73: 144-148, 2008 (In Spanish).

18. Johnson CD and Abu-Hilal M: Persistent organ failure during the first week as a marker of fatal outcome in acute pancreatitis. Gut 53: 1340-1344, 2004.

19. Buter A, Imrie CW, Carter CR, Evans S and McKay CJ: Dynamic nature of early organ dysfunction determines outcome in acute pancreatitis. Br J Surg 89: 298-302, 2002.

20. Mofidi R, Duff MD, Wigmore SJ, Madhavan KK, Garden OJ and Parks RW: Association between early systemic inflammatory response, severity of multiorgan dysfunction and death in acute pancreatitis. Br J Surg 93: 738-744, 2006. 
21. van Santvoort HC, Besselink MG, Bakker OJ, Hofker HS, Boermeester MA, Dejong CH, van Goor H, Schaapherder AF, van Eijck CH, Bollen TL, et al: A step-up approach or open necrosectomy for necrotizing pancreatitis. N Engl J Med 362: 1491-1502, 2010.

22. Petrov MS, Shanbhag S, Chakraborty M, Phillips AR and Windsor JA: Organ failure and infection of pancreatic necrosis as determinants of mortality in patients with acute pancreatitis Gastroenterology 139: 813-820, 2010.

23. Marshall JC, Cook DJ, Christou NV, Bernard GR, Sprung CL and Sibbald WJ: Multiple organ dysfunction score: A reliable descriptor of a complex clinical outcome. Crit Care Med 23: 1638-1652, 1995.

24. Balthazar EJ, Ranson JH, Naidich DP, Megibow AJ, Caccavale R and Cooper MM: Acute pancreatitis: Prognostic value of CT. Radiology 156: 767-772, 1985.

25. Mortele KJ, Wiesner W, Intriere L, Shankar S, Zou KH, Kalantari BN, Perez A, vanSonnenberg E, Ros PR, Banks PA and Silverman SG: A modified CT severity index for evaluating acute pancreatitis: Improved correlation with patient outcome. AJR Am J Roentgenol 183: 1261-1265, 2004

26. Besselink MG, van Santvoort HC, Boermeester MA, Nieuwenhuijs VB, van Goor $\mathrm{H}$, Dejong $\mathrm{CH}$, Schaapherder AF and Gooszen HG; Dutch Acute Pancreatitis Study Group: Timing and impact of infections in acute pancreatitis. Br J Surg 96: 267-273, 2009
27. Puolakkainen P, Valtonen V, Paananen A and Schröder T: C-reactive protein (CRP) and serum phospholipase A2 in the assessment of the severity of acute pancreatitis. Gut 28: 764-771, 1987.

28. Balthazar EJ: Acute pancreatitis: Assessment of severity with clinical and CT evaluation. Radiology 223: 603-613, 2002.

29. Kayar Y, Senturk H, Tozlu M, Baysal B, Atay M and Ince AT: Prediction of self-limited acute pancreatitis cases at admission to emergency unit. GE Port J Gastroenterol, 2018 doi: $10.1159 / 000493762$.

30. Tozlu M, Kayar Y, İnce AT, Baysal B and Şentürk H: Low molecular weight heparin treatment of acute moderate and severe pancreatitis: A randomized, controlled, open-label study. Turk J Gastroenterol 30: 81-87, 2019.

(c) (1) $(9$ This work is licensed under a Creative Commons c) Attribution-NonCommercial-NoDerivatives 4.0 International (CC BY-NC-ND 4.0) License. 